\title{
Missing transverse energy performance with the CMS detector
}

\author{
Florent Lacroix ${ }^{* \dagger}$ \\ UIC - Fermilab \\ E-mail: lacroixecern.ch
}

\begin{abstract}
The results of comprehensive studies of missing transverse energy as measured by the CMS detector in pp collisions at a centre-of-mass energy of $7 \mathrm{TeV}$ are presented. Three missing transverse energy reconstruction algorithms are deployed for various physics analyses. The scale and resolution for missing transverse energy are validated using vector boson and dijet events, and severe mismeasurements due to the detector are studied. We also parametrize the effects of multiple pp interactions within the same bunch crossings on the scale and resolution. A tool, called missing transverse energy significance, based on particle resolutions in each event is also presented.
\end{abstract}

The 2011 Europhysics Conference on High Energy Physics, EPS-HEP 2011,

July 21-27, 2011

Grenoble, Rhône-Alpes, France

\footnotetext{
*Speaker.

${ }^{\dagger}$ On behalf of the CMS collaboration.
} 


\section{Introduction}

Neutral weakly interacting particles, such as neutrinos, escape from typical collider detectors without producing any direct response in the detector elements. The presence of such particles must be inferred from the imbalance of total momentum. The vector momentum imbalance in the plane perpendicular to the beam direction is particularly useful in pp and $\mathrm{p} \overline{\mathrm{p}}$ colliders, and is known as missing transverse momentum, here denoted $\vec{Z}_{T}$. Its magnitude is called missing transverse energy, and is denoted $\not_{T}$.

Missing transverse energy is one of the most important observables for discriminating leptonic decays of $\mathrm{W}$ bosons and top quarks from background events which do not contain neutrinos, such as multijet and Drell-Yan events. It is also an important variable in searches for new weakly interacting, long-lived particles. Many beyond-the-standard-model scenarios, including supersymmetry, predict events with large $E_{T}$. A good understanding of large $Z_{T}$ events is very important for searches and a high-performance $\mathbb{E}_{T}$ resolution is vital for precision measurements like top or W mass measurements.

\section{Reconstruction of $E_{T}$}

In general, ${\overrightarrow{B_{T}}}_{T}$ is the negative of the vector sum of the transverse momenta of all final-state particles reconstructed in the detector. CMS has developed three distinct algorithms to reconstruct ${\overrightarrow{H_{T}}}_{T}$ : (a) $\mathrm{PF} Z_{T}$, which is calculated using a complete particle-flow technique [1]; (b) Calo $\mathbb{Z}_{T}$, which is based on calorimeter energies and the calorimeter tower geometry [2]; and (c) TC $\mathbb{F}_{T}$, which corrects Calo $Z_{T}$ by including tracks reconstructed in the inner tracker after correcting for the tracks' expected energy depositions in the calorimeter [3].

In the following, distributions involving Calo $Z_{T}$ include both jet energy scale (type-I) and unclustered energy scale (type-II) corrections [4, 5], those involving PFE ${ }_{T}$ include type-I corrections, and those involving $\mathrm{TCE}_{T}$ are uncorrected, as these were the corrections that were available at the time the analyses presented here were performed and are the versions used most typically in 2010 and 2011 physics analyses.

\section{Large $Z_{T}$ due to misreconstruction}

Various instrumental causes can lead to anomalous ${\overrightarrow{B_{T}}}_{T}$ measurements. For example, the CMS calorimeters occasionally record anomalous signals that correspond to particles hitting the transducers or produced by rare random discharges of the readout detectors. Detailed studies of the various effects are documented in detail in [5,6]. For some types of anomalous energies, the number of affected channels is small and the event can still be used in physics analysis after the removal of the anomaly. We refer to the removal process as "cleaning" the event. If a large number of channels are affected, "filters" instead tag the event as not suitable for use in physics analysis.

The basic strategy for the cleaning is based on information such as unphysical charge sharing between neighbouring channels in $\eta-\phi$ and/or depth, and timing and pulse shape information. The Calo $\not_{T}$ and $\mathrm{PF}_{T}$ distributions from minimum-bias and jet data samples are shown before and after removal of the anomalous signals in Fig. 1, demonstrating the effect of the cleaning and filters. 

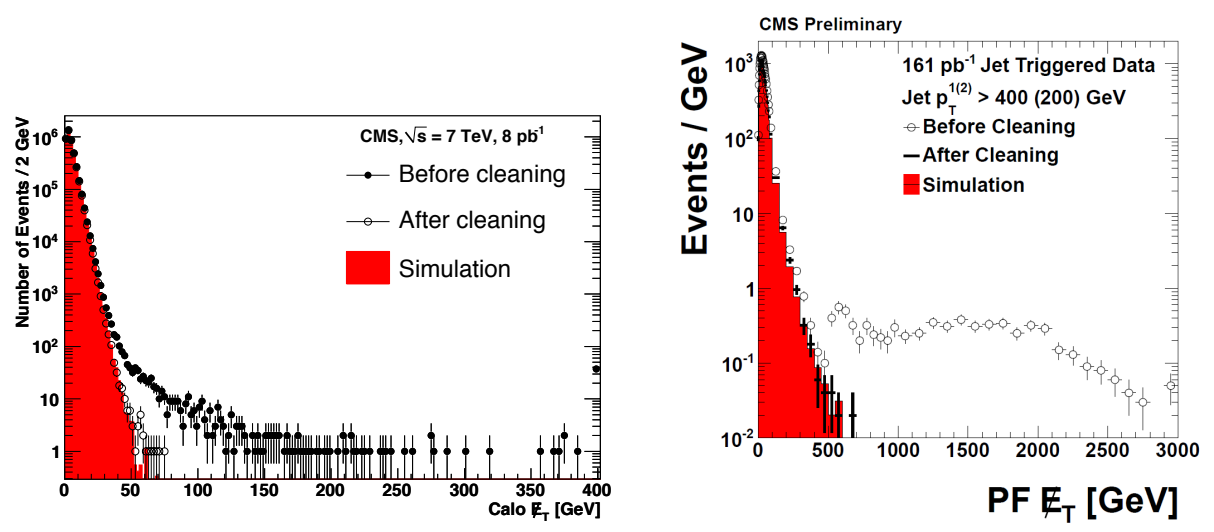

Figure 1: (left) Calo $E_{T}$ distributions in a minimum-bias data sample without (black dots) and with (open circles) cleaning and filters, compared to simulation [5]. (right) $\mathrm{PF} \mathbb{E}_{T}$ distributions in a jet data sample without (open circles) and with (black dots) cleaning and filters, compared to simulation [7].

\section{Missing transverse energy scale and resolution}

The performance of $\vec{B}_{T}$ has been studied using dijet events and events where an identified $\mathrm{Z}$ boson or isolated $\gamma$ is present, comparing the momenta of the well-measured vector boson to the hadronic recoil $[5,7]$. The agreement in response and resolutioin between the different samples is good. The measured $E_{T}$ scale agrees with the expectations of the detector simulation, but the resolution is degraded by $10 \%$ in data. As expected, the missing transverse energy reconstructed with the particle-flow algorithm gives the best performance.

The effects of multiple pp interactions within the same bunch crossings on the scale and resolution have been studied [5]. We find that pile-up interactions contribute to the degradation of the $E_{T}$ resolution but have little effect on the scale of the components of $E_{T}$. We also find that we can model its effects with a simple parametrization. The degradation of the resolution with increasing pile-up is shown in Fig. 2.

\section{5. $\not_{T}$ significance}

A spurious nonzero ${\overrightarrow{B_{T}}}_{T}$ in an event can have contributions from many sources, including measurement resolution, reconstruction inefficiencies, instrumental defects, and improper pattern recognition. Events in which the reconstructed $\vec{\not}_{T}$ is consistent with contributions solely from particle-measurement resolutions and efficiencies can be identified by evaluating the $\vec{\not}_{T}$ significance. The significance offers an event-by-event assessment of the likelihood that the observed $E_{T}$ is consistent with zero given the reconstructed content of the event and known measurement resolutions.

The computation of the $\vec{H}_{T}$ significance used in CMS is described in [5]. The performance of this variable has been studied in dijet events and in the context of a $\mathrm{W}+$ jets analysis. In dijet events, an exponential significance distribution and a flat probability distribution are obtained as expected for events with no genuine $\mathbb{E}_{T}$. This significance variable has been introduced into the selection criteria of a $\mathrm{W}+\mathrm{jets}$ analysis and a superior signal to background ratio is obtained compared to the 

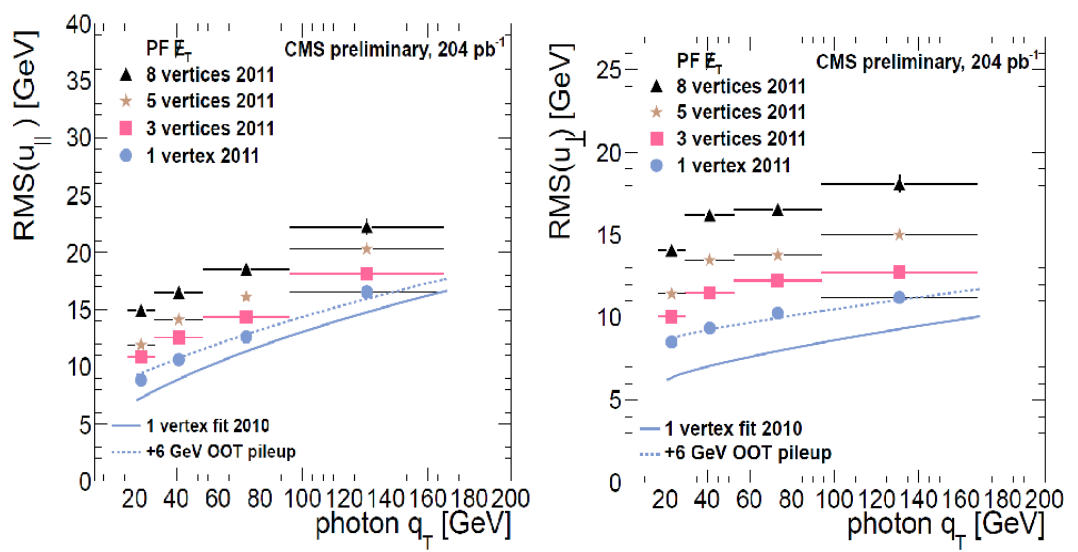

Figure 2: Resolution curves for components of hadronic recoil calculated using $\mathrm{PF} E_{T}$ in $\gamma+$ jets events, measured in events with various numbers of primary vertices, (left) parallel to the boson direction, and (right) perpendicular to the boson direction [7].

results with the traditional variables $E_{T}$ or $E_{T}$ divided by the square root of the $\sum E_{T}$, regardless of the amount of pile-up in the event [8].

\section{References}

[1] CMS Collaboration, "Particle-Flow Event Reconstruction in CMS and Performance for Jets, Taus, and $\not_{T} "$, CMS Physics Analysis Summary CMS-PAS-PFT-09-001 (2009).

[2] CMS Collaboration, "Performance of Jet Algorithms in CMS", CMS Physics Analysis Summary CMS-PAS-JME-07-003 (2007).

[3] CMS Collaboration, "Performance of Track-Corrected Missing $E_{\mathrm{T}}$ in CMS", CMS Physics Analysis Summary CMS-PAS-JME-09-010 (2009).

[4] CMS Collaboration, "Jet Performance in pp Collisions at $\sqrt{s}=7 \mathrm{TeV}$ ", CMS Physics Analysis Summary CMS-PAS-JME-10-003 (2010).

[5] CMS Collaboration, "Missing transverse energy performance of the CMS detector", JINST 6 P09001 (2011). doi:10.1088/1748-0221/6/09/P09001.

[6] CMS Collaboration, "HCAL performance from first collisions data", CMS Detector Performance Summary CMS-DP-2010-025 (2011).

CMS Collaboration, "Isolated Photon Reconstruction and Identification at $\sqrt{s}=7 \mathrm{TeV}$ ", CMS Physics Analysis Summary CMS-PAS-EGM-10-006 (2010).

CMS Collaboration, "Identification and Filtering of Uncharacteristic Noise in the CMS Hadron Calorimeter", JINST 5 T03014 (2009). doi:10.1088/1748-0221/5/03/T03014.

[7] CMS Collaboration, "MET Performance in the 2011 Data", CMS Detector Performance Summary CMS-DP-2011-010 (2011).

[8] CMS Collaboration, "Measurements of Inclusive $\mathrm{W}$ and $\mathrm{Z}$ Cross Sections in pp Collisions at $\sqrt{s}=7$ TeV", JHEP 01 (2011) 80. doi:10.1007/JHEP01(2011)080. 